\title{
On Pseudo Fuzzy COSETs of Fuzzy Normal Sub Groups
}

\author{
R.Nagarajan \\ Assistant Professor \\ Department of Mathematics \\ $\mathrm{J} \mathrm{J}$ College of Engineering \& Technology \\ Tiruchirappalli- 620009, Tamilnadu, India
}

\author{
A.Solairaju \\ Associate Professor \\ PG \& Research Department of Mathematics \\ Jamal Mohamed College \\ Tiruchirappalli- 620020, Tamilnadu, India
}

\begin{abstract}
In this paper, we have given Independent Proof of Several theorems on pseudo fuzzy cossets of fuzzy normal subgroups We investigate the notion of pseudo fuzzy double cossets, pseudo fuzzy middle cossets of a group and consider its fundamental properties. Cartesian product fuzzy relations are also discussed.
\end{abstract}

Keyword: Fuzzy groups, Fuzzy sub groups Fuzzy cossets pseudo fuzzy cosset, fuzzy pseudo middle cosset, fuzzy normal subgroup.

AMS 2000 subject classification 20N25.

\section{INTRODUCTION}

The concept of fuzzy subset of a non-empty set first was introduced by[Zadeh 1965].In [Rosenfeld 1971] formulated the fuzzification of any algebraic structures and thus opened a new directions new exploration, new path of thinking to mathematicians engineers, computer scientists and many others in various ways of various tests. The fuzzy concept is taking the vital role in all Engineering fields especially for the design and Analysis port. Mukherjee and Bhattacharya (3) introduced the fuzzy right cossets and fuzzy left cossets of a group. Here we introduce the notion of pseudo fuzzy cossets of a group and how they are related with fuzzy normal subgroups.

Definition1.1: Let $\hat{G}$ be a fuzzy set in $U$ and $\because \mathrm{G} \times$ $\mathrm{G} \rightarrow \mathrm{G}$ be a composition law, such that $(\mathrm{G}$, .) forms a group. If two conditions

$\left(\mathrm{FG}_{1}\right) \mu\left(\mathrm{gg}^{1}\right) \geq \operatorname{Min}\left\{\mu(\mathrm{g}), \mu\left(\mathrm{g}^{1}\right)\right\}$, for all $\mathrm{g}, \mathrm{g}^{1} \in \mathrm{G}$.

$\left(\mathrm{FG}_{2}\right) \mu\left(\mathrm{g}^{-1}\right)=\mu(\mathrm{g}), \mathrm{g} \in \mathrm{G}$ are satisfied, then $\hat{\mathrm{G}}$ is called a fuzzy group. If the

Supplementary condition $\left(\mathrm{FG}_{3}\right) \mu\left(\mathrm{e}_{\mathrm{G}}\right)=1$ are satisfied, then the fuzzy group is called a standardized fuzzy group where $\mathrm{e}_{\mathrm{G}}$ is an identity of the $\operatorname{group}(\mathrm{G}, \cdot)$
Definition1.2: Let ' $G$ ' be a group, a fuzzy subset ' $\mu$ ' of a group $G$ is called a fuzzy subgroup of the group $\mathrm{G}$ if

(i) $\mu(x$ y) $\geq \operatorname{Min}\{\mu(x), \mu(y)\}$, for every $x, y \in G$

(ii) $\mu\left(\mathrm{x}^{-1}\right) \geq \mu(\mathrm{x}), \mathrm{x} \in \mathrm{G}$.

\section{PROPERTIES OF FUZZY SUB GROUPS.}

2.1 Proposition: A fuzzy subset $\mu$ of a group ' $G$ ' is a fuzzy subgroup of $\hat{G}$ if and only if $\mu\left(\mathrm{x} \mathrm{y}^{-1}\right) \geq \operatorname{Min}\{\mu(\mathrm{x}), \mu(\mathrm{y})$ for every $\mathrm{x}, \mathrm{y}$ in $\mathrm{G}$ Proof: Let ' $\mu$ ' be a fuzzy subgroup of $\hat{\mathrm{G}}$. Form ' $\mu$ ' is a fuzzy group $\left(\mathrm{FG}_{1}\right)$ and $\left(\mathrm{FG}_{2}\right)$ are satisfied.

$\mu\left(\mathrm{xy}^{-1}\right) \geq \operatorname{Min}\left\{\mu(\mathrm{x}), \mu\left(\mathrm{y}^{-1}\right)\right\}=\operatorname{Min}\{\mu(\mathrm{x}) \mu(\mathrm{y})\}$ conversely let $\mu\left(\mathrm{x} \mathrm{y}^{-1}\right) \geq$

$\operatorname{Min}\{\mu(\mathrm{x}), \mu(\mathrm{y})\}$ in equality be satisfied.

Choosing $\mathrm{y}=\mathrm{x}$ we get that $\mu\left(\mathrm{xx}^{-1}\right)=\mu(\mathrm{e}) \geq \operatorname{Min}\{\mu$ $\left.(\mathrm{x}), \mu\left(\mathrm{x}^{-1}\right)\right\}=\mu(\mathrm{x})$

Hence for $\mathrm{x}=\mathrm{e}$.

$\mu\left(\mathrm{y}^{-1}\right)=\mu\left(\mathrm{ey}^{-1}\right) \geq \operatorname{Min}\{\mu(\mathrm{e}) \mu(\mathrm{y})\}=\mu(\mathrm{x})$ consequently $\mu\left(\mathrm{xy}^{-1}\right) \geq$

$\operatorname{Min}\left\{\mu(\mathrm{x}) \mu\left(\mathrm{y}^{-1}\right)\right\}=\operatorname{Min}\{\mu(\mathrm{x}), \mu(\mathrm{y})\}$

Remarks: Let ' $\mu$ ' be a fuzzy sub group of a group ' $G$ ' and $\mathrm{x} \in \mathrm{G}$. then $\mu$ (x y)= $\mu$ (y) for every $\mathrm{y} \in \mathrm{G}$ if and only if $\mu(\mathrm{x})=\mu(\mathrm{e})$

2.2 Definition : Let ' $\mu$ ' be a fuzzy sub group of a group ' $G$ '. for any $a \in G$. are defined by $(a \mu)(x)=\mu$ $\left(a^{-1} x\right)$ for every $x \in G$ is called the fuzzy cosset of the group $\mathrm{G}$ determined by ' $a$ ' and ' $\mu$ '

2.3 Definition : Let ' $\mu$ ' be the fuzzy sub group of a group $\mathrm{G}$. then for any $\mathrm{a}, \mathrm{b} \in \mathrm{G}$ a fuzzy middle cosset $\mathrm{a} \mu \mathrm{b}$ of the group $\mathrm{G}$ is defined by $(\mathrm{a} \mu \mathrm{b})(\mathrm{x})=$ $\mu\left(a^{-1} x b_{-1}\right)$ for every $x \in G$. 
2.4 Definition: Let ' $\mu$ ' be a fuzzy sub group of $\mathrm{G}$ and $\mathrm{a} \in \mathrm{G}$. Then the pseudo fuzzy cosset $(\mathrm{a}, \mu)^{\mathrm{p}}$ is defined by $(\mathrm{a} \mu) \mathrm{p}(\mathrm{x})=\mathrm{p}(\mathrm{a}) \mu(\mathrm{x})$ for every $\mathrm{x} \in \mathrm{G}$ and for some $\mathrm{p} \in \mathrm{P}$.

Example Let $\mathrm{G}=\left\{1, \mathrm{w}, \mathrm{w}^{2}\right\}$ be a group with respect to multiplication where ' $w$ ' denotes the cube root of unity . Define a map $\mu: G \rightarrow[0,1]$ by

$$
\mu(x)\left\{\begin{array}{l}
0.7 \quad \text { if } x=1 \\
=0.3 \text { if } x=w, w^{2}
\end{array}\right.
$$

the pseudo fuzzy cosset $(\mathrm{a} \mu)^{\mathrm{p}}$ for $\mathrm{p}(\mathrm{x})=0.4$ for every $\mathrm{x} \in \mathrm{G}$ to be equal to 0.28 if $\mathrm{x}=1$ and 0.12 if $\mathrm{x}=\mathrm{w}, \mathrm{w}^{2}$.

2.5 Definition: Let $\mu$ and $\lambda$ be any two fuzzy subsets of a set ' $\mathrm{X}$ ' and $\mathrm{p} \in \mathrm{P}$. the pseudo fuzzy double cosset to $(\mu \times \lambda)^{p}$ is defined as $\left((\mu x \lambda)^{p}=(x \mu)^{p} \cap(x\right.$ $\mu)^{p}$ for $x \in X$.

2.6 Definition: Let $\lambda$ and ' $\mu$ ' be two fuzzy subgroups of a group ' $G$ ' then $\lambda$ and $\mu$ are said to be Conjugate fuzzy subgroups of $\mathrm{G}$ if for some $\mathrm{g} \in \mathrm{G}$ $\lambda(\mathrm{x})=\mu\left(\mathrm{g}^{-1} \mathrm{x} g\right)$ for every $\mathrm{x} \in \mathrm{G}$.

\section{SOME PROPERTIES OF PSEUDO FUZZY COSETS.}

3.1 Proposition : Let ' $\mu$ ' be a fuzzy subgroup of a group ' $G$ ' then the pseudo fuzzy cosset $(a \mu)^{p}$ is a fuzzy sub group of ' $G$ ' for every $a \in G$.

Proof : Let ' $\mu$ ' be a fuzzy sub group of $G$, for every $\mathrm{x}, \mathrm{y}$ in $\mathrm{G}$ we have

$(\mathrm{a} \mu)^{\mathrm{p}}\left(\mathrm{xy}^{-1}\right)=\mathrm{p}(\mathrm{a}) \mu\left(\mathrm{xy}^{-1}\right) \geq \mathrm{p}$ (a) Min $\{\mu(\mathrm{x}), \mu(\mathrm{y})\}$ using (2.0)

$$
=\operatorname{Min}\{\mathrm{p}(\mathrm{a}) \mu(\mathrm{x}), \mathrm{p}(\mathrm{a}), \mu(\mathrm{y})\} \geq
$$

$\left.\operatorname{Min}\{\mathrm{a} \mu\}^{\mathrm{p}}(\mathrm{x}),(\mathrm{a}, \mu)^{\mathrm{p}}(\mathrm{y})\right\}$

for every $\mathrm{x} \in \mathrm{G}$.. this proves that $(\mathrm{a} \mu) \mathrm{p}$ is a fuzzy subgroup of $\mathrm{G}$.

Remark: A fuzzy subgroups ' $\mu$ ' of a group $G$ is said to be positive fuzzy subgroup of ' $G$ ' if ' $\mu$ ' is positive fuzzy subset of the group ' $G$ '.

3.2 Proposition : Every pseudo fuzzy double cosset is a fuzzy subgroup of a group ' $\mathrm{G}$ '

Proof: (i) $(\mu \times \lambda)^{\mathrm{p}}(\mathrm{x} y)=$

$$
\left\{(\mathrm{x} \mu)^{\mathrm{p}} \cap(\mathrm{x} \lambda)^{\mathrm{p}}\right\}(\mathrm{xy})
$$

$$
\begin{array}{r}
\left.=(\mathrm{x} \mu)^{\mathrm{p}}(\mathrm{x} y) \text { and }(\mathrm{x} \lambda)^{\mathrm{p}}(\mathrm{xy})\right\} \\
=\mathrm{p}(\mathrm{x}) \mu(\mathrm{x} y) \text { and } \mathrm{p}(\mathrm{x}) \lambda(\mathrm{xy})\}
\end{array}
$$

$\geq \mathrm{p}(\mathrm{x}) \operatorname{Min}\{\mu(\mathrm{x}), \mu(\mathrm{y})\}$ and $\mathrm{p}(\mathrm{x}) \operatorname{Min}\{\lambda(\mathrm{x}), \lambda$

(y) $\}$

$\geq \operatorname{Min}\{\mathrm{p}(\mathrm{x}) \mu(\mathrm{x}), \mathrm{p}(\mathrm{x}) \mu(\mathrm{y})\}$ and $\operatorname{Min}\{\mathrm{p}(\mathrm{x}) \lambda(\mathrm{x})$, $\mathrm{p}(\mathrm{x}) \lambda(\mathrm{y})\}$

$\geq \operatorname{Min}\{\mathrm{p}(\mathrm{x}) \mu(\mathrm{x}), \mathrm{p}(\mathrm{x}) \mu(\mathrm{x})\}, \operatorname{Min}\{\mathrm{p}(\mathrm{x}) \mu(\mathrm{y})$ and $\mathrm{p}(\mathrm{x}) \lambda(\mathrm{y})\}$

$\left.\left.=\operatorname{Min}\left\{(\mathrm{x} \mu)^{\mathrm{p}} \cap(\mathrm{x} \lambda)^{\mathrm{p}}\right\}(\mathrm{x}),(\mathrm{x} \mu)^{\mathrm{p}} \mathrm{n}(\mathrm{x} \lambda)^{\mathrm{p}}\right)(\mathrm{y})\right\}$

$\geq \operatorname{Min}\left\{(\mu \mathrm{x} \lambda)^{\mathrm{p}}(\mathrm{x}),(\mu \mathrm{x} \lambda)^{\mathrm{p}}(\mathrm{y})\right\}$

(ii) $(\mu \mathrm{x} \lambda)^{\mathrm{p}}(\mathrm{x})=\left\{(\mathrm{x} \mu)^{\mathrm{p}} \cap(\mathrm{x} \lambda)^{\mathrm{p}}\right\}(\mathrm{x})$

$$
=(\mathrm{x} \mu)^{\mathrm{p}} \cap(\mathrm{x} \lambda)^{\mathrm{p}}(\mathrm{x})
$$

$=\mathrm{p}(\mathrm{x}) \mu(\mathrm{x})$ and $\mathrm{p}(\mathrm{x}) \lambda(\mathrm{x})$

$=\mathrm{p}(\mathrm{x}) \mu(\mathrm{x})^{-1}$ and $\mathrm{p}(\mathrm{x}) \lambda(\mathrm{x})^{-1}$ (since $\lambda$

and $\mu$ are fuzzy subsets)

$$
\begin{aligned}
& =(\mathrm{x} \mu)^{\mathrm{p}}(\mathrm{x})^{-1} \text { and }(\mathrm{x} \lambda)^{\mathrm{p}}(\mathrm{x})^{-1} \\
= & \left\{(\mathrm{x} \mu)^{\mathrm{p}} \mathrm{n}(\mathrm{x} \lambda)^{\mathrm{p}}\right\}(\mathrm{x})^{-1} \\
= & (\mu \mathrm{x} \lambda)^{\mathrm{p}}(\mathrm{x})^{-1}
\end{aligned}
$$

Theorem is proved.

3.3 Proposition : Every fuzzy middle cosset of a group ' $G$ ' is a fuzzy subgroup of $G$.

Proof : Let a $\mu$ b be a fuzzy middle cosset of the group ' $G$ ' and ' $\lambda$ ' and ' $\mu$ ' be two conjugate fuzzy subgroups of $\mathrm{G}$.

(i) $(\mathrm{a} \mu \mathrm{b})(\mathrm{x} y)=\mu\left(\mathrm{a}^{-1} \mathrm{x} \mathrm{y}^{-1}\right)$ $=\lambda(\mathrm{x} \mathrm{y})[\because: \lambda$ and $\mu$ conjugate

fuzzy subgroups]

$$
\geq \operatorname{Min}\{\lambda(\mathrm{x}), \lambda(\mathrm{y})\}
$$

$\geq \operatorname{Min}\left\{\mu\left(\mathrm{a}^{-1} \mathrm{xb}^{-1}\right), \mu\left(\left(\mathrm{a}^{-1} \mathrm{yb}^{-1}\right)\right\}\right.$

$\geq \operatorname{Min}\{(\mathrm{a} \mu \mathrm{b})(\mathrm{x}),(\mathrm{a} \mu \mathrm{b})(\mathrm{y})\}$

(ii) $(\mathrm{a} \mu \mathrm{b})(\mathrm{x}) \quad=\mu\left(\mathrm{a}^{-1} \mathrm{x} \mathrm{b}^{-1}\right)$

$=\mu\left(a^{-1} x^{-1} b^{-1}\right)(\cdot:$ ' $\mu$ ' fuzzy sub group $)$

$=(\mathrm{a} \mu \mathrm{b})\left(\mathrm{x}^{-1}\right)$ Theorem is proved.

3.4 Definition : Let ' $G$ ' be a group. A fuzzy subgroup ' $\mu$ ' of ' $G$ ' is called normal if $\mu(x)=\mu\left(y^{-1} x\right.$ y) for all $\mathrm{x}, \mathrm{y}$ in $\mathrm{G}$. (or) A fuzzy subgroup $\mu_{\mathrm{H}}$ of $\mathrm{G}$ is called a fuzzy normal subgroup of ' $G$ ' if $\mu_{H}$ ( $x$ y) $=\mu_{H}$ ( y $\mathrm{x}$ ) for all $\mathrm{x}, \mathrm{y}$ in $\mathrm{G}$.

3.5 Proposition: Every pseudo fuzzy cosset is a fuzzy normal subgroup of a group ' $G$ '

Proof : Let $(a \mu)^{p}$ be any pseudo fuzzy cosset. $a \in G$ and for some $\mathrm{p} \in \mathrm{P}$. Now 


$$
\begin{aligned}
& \begin{aligned}
(\mathrm{a} \mu)^{\mathrm{p}}(\mathrm{x}) & =\mathrm{p}(\mathrm{a}) \mu(\mathrm{x}) \\
= & \mathrm{p}(\mathrm{a}) \operatorname{Min}\{\mu(\mathrm{e}), \mu(\mathrm{x})\} \\
= & \mathrm{p}(\mathrm{a}) \operatorname{Min}\left\{\mu\left(\mathrm{y}^{-1} \mathrm{y}\right), \mu(\mathrm{x})\right\}
\end{aligned} \\
& \geq \mathrm{p} \text { (a) Min }\left\{\operatorname{Min}\left\{\mu(\mathrm{y})^{-1,} \mu(\mathrm{y})\right\}, \mu(\mathrm{x})\right\} \\
& \geq \mathrm{p} \text { (a) } \operatorname{Min}\left\{\mu(\mathrm{y})^{-1}, \operatorname{Min}\{\mu(\mathrm{y}), \mu(\mathrm{x})\}\right. \\
& =\mathrm{p} \text { (a) } \mu\left(\mathrm{y}^{-1} \mathrm{x} y\right) \text { for all } \mathrm{y} \in \mathrm{G} .
\end{aligned}
$$

Aliter: Let $(\mathrm{a} \mu)^{\mathrm{p}}$ be any pseudo fuzzy cosset and a $\in G$ for some $p \in P$, Let $\mu_{H}$ is a fuzzy normal subgroup of $\mathrm{G}$.

Now $(\text { a } \mu \mathrm{H})^{\mathrm{p}}(\mathrm{x}$ y $)=\mathrm{p}(\mathrm{a}) \mu_{\mathrm{H}}(\mathrm{xy})$

fuzzy normal)

$$
=\mathrm{p} \text { (a) } \mu_{\mathrm{H}}(\mathrm{yx})\left(\mu_{\mathrm{H}}\right. \text { is }
$$

$$
=\left(\mathrm{a} \mu_{\mathrm{H}}\right)^{\mathrm{p}}(\mathrm{y} \mathrm{x})
$$

3.6 Proposition: the intersection of two pseudo fuzzy cosset normal subgroup is also fuzzy normal subgroup of a group.

Proof: Let $(\mathrm{a} \mu)^{\mathrm{p}}$ and $(\mathrm{b} \mu)^{\mathrm{p}}$ be any two pseudo fuzzy cosset normal subgroup of $\mathrm{G}$.

$(\mathrm{a} \mu)^{\mathrm{p}}(\mathrm{x})=\left(\mathrm{a} \mu^{\mathrm{p}}\left(\mathrm{y}^{-1} \mathrm{x} y\right), \mathrm{y} \in \mathrm{G}---(1)\right.$

$(\mathrm{b} \mu)^{\mathrm{p}}(\mathrm{x})=(\mathrm{a} \mu)^{\mathrm{p}}\left(\mathrm{y}^{-1} \mathrm{x} \mathrm{y}\right), \mathrm{y} \in \mathrm{G}-(2)$

Now

$$
\begin{aligned}
\left\{(\mathrm{a} \mu)^{\mathrm{p}} \cap(\mathrm{b} \mu)^{\mathrm{p}}(\mathrm{x})=((\mathrm{a} \cap \mathrm{b}) \mu)^{\mathrm{p}}(\mathrm{x})\right. \\
=\mathrm{p}(\mathrm{a} \cap \mathrm{b}) \mu(\mathrm{x}) \\
=\mathrm{p}(\mathrm{a}) \cdot \mathrm{p}(\mathrm{b}) \mu(\mathrm{x})
\end{aligned}
$$

$=\mathrm{p}(\mathrm{a}) \cdot \mu(\mathrm{x})$ and $\mathrm{p}(\mathrm{b}) \mu(\mathrm{x})$

$=(\mathrm{a} \mu)^{\mathrm{p}}(\mathrm{x})$ and $(\mathrm{b} \mu)^{\mathrm{p}}(\mathrm{x})$

$=(\mathrm{a} \mu)^{\mathrm{p}}\left(\mathrm{y}^{-1} \mathrm{x} \mathrm{y}\right)$ and $(\mathrm{b} \mu)^{\mathrm{p}}\left(\mathrm{y}^{-1} \mathrm{x}\right.$ y) by $((\mathrm{i}) \&(\mathrm{ii}))$

$=\mathrm{p}(\mathrm{a}) \cdot \mathrm{p}(\mathrm{b}) \mu\left(\mathrm{y}^{-1} \mathrm{x} y\right)$

$=((\mathrm{a} \cap \mathrm{b}) \mu)^{\mathrm{p}}\left(\mathrm{y}^{-1} \mathrm{xy}\right)$

$=\left\{(\mathrm{a} \mu)^{\mathrm{p}} \cap(\mathrm{b} \mu)^{\mathrm{p}}\right\}\left(\mathrm{y}^{-1} \mathrm{x} \mathrm{y}\right)$. Theorem is proved

Aliter: Let $\left.\left(\mathrm{a} \mu_{\mathrm{H}}\right)^{\mathrm{p}} \cap\left(\mathrm{b} \mu_{\mathrm{H}}\right)^{\mathrm{p}}\right\}(\mathrm{x} y)=$ $\left.\left((\mathrm{a} \cap \mathrm{b}) \mu_{\mathrm{H}}\right)^{\mathrm{p}}\right\}(\mathrm{x} y)$

$=\mathrm{p}(\mathrm{a} \cap \mathrm{b}) \mu$

(x y) $\quad=\mathrm{p}(\mathrm{a} \cap \mathrm{b}) \mu_{\mathrm{H}}(\mathrm{y} x)$

( $\mu_{\mathrm{H}}$ is fuzzy normal)

$$
\left.\left.=(\mathrm{a} \cap \mathrm{b}) \mu_{\mathrm{H}}\right)^{\mathrm{p}}\right\}(\mathrm{yx})=\{\mathrm{a} \cap \mathrm{b})
$$

$\left.{ }^{\mathrm{p}} \cap\left(\mathrm{b} \mu_{\mathrm{H}}\right)^{\mathrm{p}}\right\}(\mathrm{y} \mathrm{x})$

3.7 : Proposition : Pseudo fuzzy double cosset is a fuzzy normal subgroup of a group ' $G$ '

Proof: Let $(\mu \times \lambda)^{p}$ be any pseudo fuzzy double cosset for $\mathrm{x} \in \mathrm{X}$.

Now

$$
\begin{gathered}
(\mu \mathrm{x} \lambda)^{\mathrm{p}}(\mathrm{x}) \quad=\left\{(\mathrm{x} \mu)^{\mathrm{p}} \cap(\mathrm{x} \lambda)^{\mathrm{p}}\right\}(\mathrm{x}) \\
\left.=(\mathrm{x} \mu)^{\mathrm{p}}(\mathrm{x}) \cap(\mathrm{x} \lambda)^{\mathrm{p}}\right\}(\mathrm{x})
\end{gathered}
$$

$$
\begin{aligned}
& =\mathrm{p}(\mathrm{x}) \cap \mu(\mathrm{x}) \cap \mathrm{p}(\mathrm{x}) \lambda(\mathrm{x}) \\
& =\mathrm{p}(\mathrm{x}) \operatorname{Min}\{\mu(\mathrm{x}), \mu(\mathrm{e})\} \cap \mathrm{p}(\mathrm{x}) \operatorname{Min}\{\lambda
\end{aligned}
$$

$(\mathrm{x}), \lambda(\mathrm{e})\}$

$$
=\mathrm{p}(\mathrm{x}) \operatorname{Min}\left\{\mu \quad(\mathrm{x}), \mu\left(\mathrm{y}^{-1} \mathrm{y}\right)\right\} \cap \mathrm{p}(\mathrm{x})
$$

$\operatorname{Min}\left\{\lambda(\mathrm{x}), \lambda\left(\mathrm{y}^{-1} \mathrm{y}^{1}\right)\right.$

$$
\geq \mathrm{p}(\mathrm{x}) \operatorname{Min}\left\{\mu \quad(\mathrm{x}), \operatorname{Min} \mu\left(\mathrm{y}^{-1}\right), \mu(\mathrm{y})\right\}
$$

$\cap$

$\mathrm{p}(\mathrm{x}) \operatorname{Min}\left\{\lambda(\mathrm{x}), \operatorname{Min}\left\{\lambda\left(\mathrm{y}^{-1}\right), \lambda(\mathrm{y})\right\}\right.$

$=\mathrm{p}(\mathrm{x}) \operatorname{Min}\left\{\mu\left(\mathrm{y}^{-1}\right), \mu(\mathrm{x} y)\right\} \cap \mathrm{p}(\mathrm{x}) \operatorname{Min}\left\{\lambda\left(\mathrm{y}^{-1}\right), \lambda\right.$ (x y) $\}$

$=\mathrm{p}(\mathrm{x}) \quad \mu\left(\mathrm{y}^{-1} \mathrm{x} y\right) \cap \mathrm{p}(\mathrm{x}) \lambda\left(\mathrm{y}^{-1} \mathrm{x} \mathrm{y}\right)$

$\left.=\{\mathrm{x} \mu\}^{\mathrm{p}} \cap(\mathrm{x} \lambda)^{\mathrm{p}}\right\}\left(\mathrm{y}^{-1} \mathrm{x} \mathrm{y}\right)$

$=(\mu \times \lambda)^{p}\left(y^{-1} \mathrm{x} y\right)$ Theorem is proved.

3.8 Proposition: Fuzzy middle cossets forms a fuzzy normal subgroup of $\mathrm{G}$.

Proof: $(\mathrm{a} \mu \mathrm{b})(\mathrm{x})=\mu\left(\mathrm{a}^{-1} \mathrm{x} \mathrm{b}^{-1}\right)=\lambda(\mathrm{x})$

$$
\begin{aligned}
& =\operatorname{Min}\{\lambda(\mathrm{x}), \lambda(\mathrm{e})\} \\
& =\operatorname{Min}\left\{\lambda(\mathrm{x}), \lambda\left(\mathrm{y}^{-1} \mathrm{y}\right)\right\} \\
\geq & \operatorname{Min}\left\{\lambda(\mathrm{x}), \operatorname{Min}\left(\lambda\left(\mathrm{y}^{-1}\right), \lambda(\mathrm{y})\right)\right\} \\
= & \operatorname{Min}\left\{\lambda\left(\mathrm{y}^{-1}\right) \operatorname{Min}(\lambda(\mathrm{x}), \lambda(\mathrm{y})\}\right. \\
= & \operatorname{Min}\left(\lambda\left(\mathrm{y}^{-1}\right), \lambda(\mathrm{x} y)\right\}=\lambda\left(\mathrm{y}^{-1} \mathrm{x}\right) \\
= & \mu\left(\mathrm{a}^{-1}\left(\mathrm{y}^{-1} \mathrm{x} y\right) \mathrm{b}^{-1}\right)=(\mathrm{a} \mu \mathrm{b})\left(\mathrm{y}^{-1} \mathrm{x} y\right)
\end{aligned}
$$

3.9 Definition: The strong Fuzzy $\alpha$-cut is defined as $\mathrm{A}_{\alpha}^{+}=\{\mathrm{x} / \mathrm{A}(\mathrm{x})>\alpha\}$ where $\mathrm{A}$ is any Fuzzy set

3.10 Definition: Let ' $A$ ' be a Fuzzy set in a set $S$, the strongest Fuzzy relation on ' $S$ ' (ie) Fuzzy relation on ' $\mathrm{A}$ ' is $\mu_{\mathrm{A}}(\mathrm{x}, \mathrm{y})=\operatorname{Min}\{(\mathrm{A}(\mathrm{x}), \mathrm{A}(\mathrm{y})\}$

3.11 Definition: Cartesian Product: Let $\lambda$ and $\mu$ be any two Fuzzy sets in $\mathrm{X}$ then the Cartesian Product of $\lambda$ and $\mu$ is $\lambda x \mu: x \times x \rightarrow[0,1]$ defined by $(\lambda \times \mu)(x, y)=$ Min $\{\lambda(\mathrm{x}), \mu(\mathrm{y})\}$ for all $\mathrm{x}, \mathrm{y} \varepsilon \mathrm{X}$.

3.12 Proposition: Let $\mu_{\mathrm{A}}$ be a strongest Fuzzy relation on ' $\mathrm{S}$ ' and ' $\mathrm{A}_{\alpha}^{+}$' be the strong $\alpha$-cut the $\mu_{\mathrm{A}}$ forms a strong $\alpha$-cut Fuzzy group on $\mathrm{S}$.

Proof: Let A:S $\rightarrow[0,1]$ be any function and $\mu_{\mathrm{A}}$ be the strongest Fuzzy relation on $\mathrm{S}$.

(i) Let $x, y \in S$

$$
\begin{aligned}
\mu_{\mathrm{A}}(\mathrm{x}, \mathrm{y}) & =\operatorname{Min}\{\mathrm{A}(\mathrm{x}), \mathrm{A}(\mathrm{y})\} \\
& \geq \operatorname{Min}\{\alpha, \alpha\} \\
\mu_{\mathrm{A}}(\mathrm{x}, \mathrm{y}) & \geq \quad \alpha \\
\text { (ii) } \mu_{\mathrm{A}}\left(\mathrm{x}^{-1}, \mathrm{y}^{-1}\right) & =\operatorname{Min}\left\{\mathrm{A}\left(\mathrm{x}^{-1}\right), \mathrm{A}\left(\mathrm{y}^{-1}\right)\right\} \\
= & \operatorname{Min}\{\mathrm{A}(\mathrm{x}), \mathrm{A}(\mathrm{y})\} \\
& =\mu_{\mathrm{A}}(\mathrm{x}, \mathrm{y})
\end{aligned}
$$


(ii) $\mu_{\mathrm{A}}(\mathrm{e}, \mathrm{e}) \quad=\operatorname{Min}\{\mathrm{A}(\mathrm{e}), \mathrm{A}(\mathrm{e})\}$

$=\operatorname{Min}\{1,1\}$

$=1$

$\mu_{\mathrm{A}}$ Forms a strong Fuzzy group $\alpha$ - cut on $\mathrm{S}$.

3..13 Proposition: Let $\lambda$ and $\mu$ be strong Fuzzy $\alpha$ cuts on $\mathrm{S}$ then $\lambda \times \mu$ is a strong Fuzzy group $\alpha$ - cut.

Proof: Let $\mathrm{x}, \mathrm{y} \varepsilon \mathrm{S}$ and $\lambda: \mathrm{x} \times \mathrm{x} \rightarrow[0,1]$ be any function.

$\begin{array}{rlrl}\text { i. }(\lambda \times \mu)(\mathrm{x}, \mathrm{y}) & =\operatorname{Min}\{\lambda(\mathrm{x}), \mu(\mathrm{y})\} \\ & \geq & \operatorname{Min}\{\alpha, \alpha\} \\ & \geq & \alpha \\ \text { (ii) }(\lambda \times \mu)\left(\mathrm{x}^{-1}, \mathrm{y}^{-1}\right)= & \operatorname{Min}\left\{\lambda\left(\mathrm{x}^{-1}\right), \mu\left(\mathrm{y}^{-1}\right)\right\} \\ & = & \operatorname{Min}\{\mathrm{A}(\mathrm{x}), \mathrm{A}(\mathrm{y})\} \\ & = & (\lambda \times \mu)(\mathrm{x}, \mathrm{y}) \\ \text { (ii) }(\lambda \times \mu)(\mathrm{e}, \mathrm{e})= & \operatorname{Min}\{\lambda(\mathrm{e}), \mu(\mathrm{e})\} \\ & = & \operatorname{Min}\{1,1\} \\ & = & 1\end{array}$

$(\lambda \times \mu)$ forms a strong Fuzzy group $\alpha$-cut on $S$.

Remark : i) Min $(a, b)^{i}=\operatorname{Min}\left\{a^{i}, b^{i}\right)$ for all Positive integer 'i'\}

ii) $\mu_{\mathrm{A}} \mathrm{i}(\mathrm{x}, \mathrm{y})=\quad\left(\mu_{\mathrm{A}}(\mathrm{x}, \mathrm{y})\right)^{\mathrm{i}}$

$=\operatorname{Min}\{\mathrm{A}(\mathrm{x}), \mathrm{A}(\mathrm{y})\}^{\mathrm{i}}$

$=\operatorname{Min}\left\{\mathrm{A}^{\mathrm{i}}(\mathrm{x}), \mathrm{A}^{\mathrm{i}}(\mathrm{y})\right\}$

3.14 Proposition : Let $\mu_{A}{ }^{i}$ and $\mu_{A}{ }^{j}$ be two strong Fuzzy relations and $\mathrm{A}_{\alpha}^{+}$be strong Fuzzy $\alpha$ - cut then $\mu_{A}{ }^{i}{ }_{U A}^{j}$ forms a strong Fuzzy $\alpha$-cut on $S$.

Proof: Since $\mathrm{i}<\mathrm{j}$

$\mu_{\mathrm{A} U A^{\mathrm{i}}}{ }^{\mathrm{j}}(\mathrm{x}, \mathrm{y})=\left\{\left(\mathrm{A}^{\mathrm{i}} \mathrm{UA}^{\mathrm{j}},\right)(\mathrm{x}),\left(\mathrm{A}^{\mathrm{i}} \mathrm{U}^{\mathrm{j}}\right)(\mathrm{y})\right\}$

$=\operatorname{Min} \quad\left\{\operatorname{Max}\left\{\mathrm{A}^{\mathrm{i}}(\mathrm{x}), \quad \mathrm{A}^{\mathrm{j}}(\mathrm{x})\right\}, \quad \operatorname{Max}\right.$

$\left\{\mathrm{A}^{\mathrm{i}}(\mathrm{y}), \quad \mathrm{A}^{\mathrm{j}}(\mathrm{y})\right\}$

$=\operatorname{Max} \quad\left\{\operatorname{Min} \quad\left\{\mathrm{A}^{\mathrm{i}}(\mathrm{x}), \quad \mathrm{A}^{\mathrm{i}}(\mathrm{y})\right\}, \quad \operatorname{Min} \quad\left\{\mathrm{A}^{\mathrm{j}}(\mathrm{x}), \quad \mathrm{A}^{\mathrm{j}}(\mathrm{y})\right\}\right.$

$=\operatorname{Max} \quad\left\{\operatorname{Min} \quad\{\mathrm{A}(\mathrm{x}), \quad \mathrm{A}(\mathrm{y})\}^{\mathrm{I}}, \operatorname{Min} \quad\{\mathrm{A}(\mathrm{x}), \quad \mathrm{A}(\mathrm{y})\}^{\mathrm{j}}\right\}$

$\geq \operatorname{Max}\left\{\operatorname{Min}\{\alpha, \alpha\}^{\mathrm{i}}, \operatorname{Min}\{\alpha, \alpha\}^{\mathrm{j}}\right\}$

$\geq \operatorname{Max}\left\{\operatorname{Min}\left\{\alpha^{\mathrm{i}}, \alpha^{\mathrm{i}}\right\}, \operatorname{Min}\left\{\alpha^{\mathrm{j}}, \alpha^{\mathrm{j}}\right\}\right\}$

$\geq \operatorname{Max}\left\{\alpha^{\mathrm{i}}, \alpha^{\mathrm{j}}\right\}$

$\geq \alpha^{\mathrm{i}}$

$\mu_{A}{ }^{i}{ }^{j}{ }^{j}$ is a strong fuzzy $\alpha$-cut on $S$.

REMARK Let $\mu_{\mathrm{A}}{ }^{\mathrm{i}}$ and $\mu_{\mathrm{A}}{ }^{\mathrm{j}}$ be two strong Fuzzy relations and $\mathrm{A}_{\alpha}{ }^{+}$be strong fuzzy $\alpha$-cut $\mu_{\mathrm{A}}{ }^{\mathrm{i}}{ }_{\mathrm{nA}}{ }^{\mathrm{j}}$ is a strong fuzzy $\alpha$-cut on $\mathrm{S}$.

Proof: It is obvious

Definition 3.15 : A fuzzy binary relation $\mu$ on a semi group ' $S$ ' is called fuzzy compatible iff $\mu$ (ac, bd) $\geq$ $\operatorname{Min}\{\mu(a, b), \mu(c, d)\}$ for all a,b,c,d $\varepsilon S$.
3.16 Preposition: Let $\mu_{\mathrm{A}}$ be the strongest Fuzzy

Relation on $\mathrm{S}$ then $\mathrm{A}_{\alpha}{ }^{+}$is a strong $\alpha$-cut then $\mu_{\mathrm{A}}$ forms a Fuzzy compatible.

Proof:

Now $\mu_{\mathrm{A}}(\mathrm{ac}, \mathrm{bd})=\operatorname{Min}\{\mathrm{A}(\mathrm{ac}),(\mathrm{A}(\mathrm{bd})\}$

$\geq \operatorname{Min}\{\operatorname{Min}\{\mathrm{A}(\mathrm{a}), \mathrm{A}(\mathrm{c})\}, \operatorname{Min}(\mathrm{A}(\mathrm{b}) \mathrm{A}(\mathrm{d})\}$

$\geq \operatorname{Min}\left\{\mu_{\mathrm{A}}(\mathrm{a}, \mathrm{b}), \mu_{\mathrm{A}}(\mathrm{b}, \mathrm{d})\right\}$

3.18 Preposition: Let $\mu_{\mathrm{A}}$ be a fuzzy compatible then it forms a strong fuzzy

$\alpha$-cut

Proof:

Now $\mu_{\mathrm{A}}(\mathrm{ac}, \mathrm{bd}) \geq\left\{\mu_{\mathrm{A}}(\mathrm{a}, \mathrm{b}), \mu_{\mathrm{A}}(\mathrm{b}, \mathrm{d})\right\}$

$=\operatorname{Min}\{\operatorname{Min}(\mathrm{A}(\mathrm{a}), \mathrm{A}(\mathrm{b})\}, \operatorname{Min}\{\mathrm{A}(\mathrm{b}), \mathrm{A}(\mathrm{d})\}\}$

$>\operatorname{Min}\{\operatorname{Min}\{\alpha, \alpha\}, \operatorname{Min}\{\alpha, \alpha\}\}$

$>\operatorname{Min}\{\alpha, \alpha\}$

$>\alpha$

Fuzzy Compatible forms a strong fuzzy $\alpha$-cut

\section{References}

[1]K.A. Dib and A.M. Hassan, the fuzzy normal subgroups, Fuzzy cossets and systems 98 (1988), 393402.

[2]KUROKI, N. Fuzzy congruencies and fuzzy normal subgroups, in form. Su. 60, 247 - 259 (1992)

[3]N.P. Mukherjee and Prabir Bhattacharya, Fuzzy normal subgroups and fuzzy cossets, inform Sci.34, 225-239

[4]MORSI, Nehad N., Note on " Normal fuzzy subgroups and fuzzy normal series of finite groups", Fuzzy sets and systems, 87, 255-256 (1997)

[5]A Rosenfeld, Fuzzy groups, J.Math. Anal. Appl. 35 (1971), 512 -517.

[6]Wanging Wu, Normal fuzzy groups, fuzzy math I (1), (1981) 21-30

[7]L.A. Zadeh, fuzzy sets, inform and control 8 (1965) 338-365. 